\title{
Collaboratively charting the gene-to-phenotype network of human congenital heart defects
}

\author{
Roland Barriot ${ }^{1,2,3 \dagger}$, Jeroen Breckpott ${ }^{4 \dagger}$, Bernard Thienpont ${ }^{4,5 \dagger}$, Sylvain Brohée ${ }^{1 \dagger}$, Steven Van Vooren', Bert Coessens', \\ Leon-Charles Tranchevent', Peter Van Loo ${ }^{1,4,6}$, Marc Gewillig7, Koenraad Devriendt ${ }^{1 \neq}$ and Yves Moreau'** \\ CHDWiki is accessible at http://www.esat.kuleuven.be/ bioiuser/chdwiki
}

\begin{abstract}
Background: How to efficiently integrate the daily practice of molecular biologists, geneticists, and clinicians with the emerging computational strategies from systems biology is still much of an open question.

Description: We built on the recent advances in Wiki-based technologies to develop a collaborative knowledge base and gene prioritization portal aimed at mapping genes and genomic regions, and untangling their relations with corresponding human phenotypes, congenital heart defects (CHDs). This portal is not only an evolving community repository of current knowledge on the genetic basis of CHDs, but also a collaborative environment for the study of candidate genes potentially implicated in CHDs - in particular by integrating recent strategies for the statistical prioritization of candidate genes. It thus serves and connects the broad community that is facing CHDs, ranging from the pediatric cardiologist and clinical geneticist to the basic investigator of cardiogenesis.

Conclusions: This study describes the first specialized portal to collaboratively annotate and analyze gene-phenotype networks. Of broad interest to the biological community, we argue that such portals will play a significant role in systems biology studies of numerous complex biological processes.
\end{abstract}

\section{Background}

Recently, Wiki technology - inspired by the well-known Wikipedia encyclopedia - has been proposed as a potential strategy for the collaborative development of biological knowledge bases [1-6]. Although a 'Wikipedia for Genes' is likely to emerge, a number of challenges remain. First, classical Wiki technology in itself (based on free text) is unsuitable for developing genetic knowledge bases because of the imperative need for structured information. Hence, Wiki platforms for genetic knowledge bases need to provide a strong framework for integration with classical database technology. Wikiproteins already implements this need at a high level by abstractly linking concepts, such as proteins and biological processes [1]. Second, and probably foremost, each

${ }^{\dagger}$ Contributed equally

${ }^{\ddagger}$ Joint senior authors

*Correspondence: yves.moreau@esat.kuleuven.be

'Bioinformatics Group, Department of Electrical Engineering, ESAT-SCD, Katholieke Universiteit Leuven, Kasteelpark Arenberg 10, B-3001 Leuven, Belgium

Full list of author information is available at the end of the article community uses specific terminology, has specific goals, and uses specific data and tools. Such specificity cannot be addressed in a generic Wikipedia for Genes and requires tailored solutions implementing different levels of specialization. Third, Wiki technology does not in itself support downstream analysis of the information gathered in the Wiki.

Going beyond knowledge gathering, integrative data analysis strategies have been proposed recently for the prioritization of genes potentially involved in a given biological process, phenotype, or disease [7-9]. Nevertheless, there is clearly a gap between such advanced (and somewhat complex) analysis strategies and actual wet lab work. A similar gap can be observed between those strategies and clinical genetics where increasingly complex molecular data need to be interpreted towards the diagnosis of constitutional disorders. To bridge this gap and bring integrative analysis strategies into practice, we integrate a candidate gene prioritization method [9] and browsing of networks of gene interactions [10-12] into the Wiki platform. 
We therefore propose to combine Wiki technology, databases of genomic and phenomic information, and data analysis tools into a Wiki portal that supports the needs of a specialized community. In particular, we describe a Wiki portal for the genetic study of congenital heart defects (CHDs), termed CHDWiki. CHDs are the major cause of mortality in newborns in the developed world, but despite this manifest importance, most CHDs still have unknown etiologies. In some instances, specific genetic and environmental factors have been shown to cause CHDs. A review of the etiology of CHDs is available through CHDWiki [13].

The CHDWiki portal focuses on mapping out the gene network leading to human CHD phenotypes. It supports both genetic and molecular biology research that aims at hunting for CHD genes, as well as clinical research that aims at identifying and interpreting genetic aberrations in patients suffering from well-characterized CHDs.

\section{Construction and content Knowledge acquisition}

To build a set of most currently known gene-phenotype links, OMIM (Online Mendelian Inheritance in Man) and MEDLINE were manually searched by an expert in the field for genes that are linked with any of 139 relevant cardiac defect phenotypes listed among the internationally used CHD codes from the Association for European Paediatric Cardiology (AEPC). The use of this specialized ontology maximizes the relevance of the collected information to the CHD community and improves the consistency of this information. Relevant genes and mutations were selected and their corresponding cardiac phenotype were manually gathered and described based on the available literature. The level of support for a gene-phenotype link was defined by its incidence and the number of independent publications reporting it. We only considered such links confirmed if at least two reports from independent groups described the incidence of CHD in patients with a mutation to be greater than $1 \%$. Moreover, the support for the link between every single gene mutation and CHD type was further characterized based on the genetic evidence (inheritance and incidence), in silico predictions, and the functional studies (in vitro analysis and animal models) described in the study.

To build a set of most currently known chromosomal regions linked to CHDs, MEDLINE was searched for imbalances detected by molecular karyotyping, breakpoints of balanced chromosome aberrations or regions implicated in CHDs through linkage studies. These data complement at a much higher resolution the CHD regions identified by Van Karnebeek et al. [14], which were based on reported cytogenetically visible chromosomal aberrations.
CHDWiki is conceived to allow straightforward inclusion of published and unpublished data from all collaborators. All clinical (cardiac and non-cardiac) and molecular data are rendered anonymous and collected in a standardized manner, either directly in the CHDWiki database (for genes, translocation breakpoints and linkage regions) or (for well-delineated chromosomal imbalances) using another tool designed for this purpose, CHDBench [15]. Consent for submitting unpublished data from a patient or their legal representative is explicitly required, and was assumed to be obtained for published data that are included. Ethical approval for the incorporation of patient data was obtained from the Ethics Committee KU Leuven (S51093).

\section{Platform development}

CHDWiki is based on the MediaWiki engine initially developed for the Wikipedia project. We implemented a generic extension that allows registering specific components for the management of structured data and for the on-the-fly execution of analysis tools.

The benefits of databases are manifold and become apparent when providing different views on the same data. For instance, it allows providing the detailed list of genes linked to CHDs, as well as the list of CHDs having linked genes. Databases also solve consistency issues; for example, when a link is added or updated between a gene and a specific CHD, both the gene page and the CHD page instantaneously reflect this change. The principles of the generic extensions are the following. Specific components can subscribe to pages so that the Wiki engine executes these components when the page is rendered, or they can explicitly be called from within the page through the use of a specific tag.

For example, to include the list of CHDs linked to $J A G 1$, the generic extension first calls the chdsForGenes component with parameter JAG1 to retrieve the data in the form of (variable, value) pairs. Second, the extension retrieves the chdsForGeneTemplate layout template, which is stored as Wiki text in a standard Wiki page. Third, it replaces the variables by their actual values. Eventually, the resulting Wiki text is rendered by the MediaWiki engine.

The simplicity of the generic extension mechanism makes it both flexible and powerful. Specific components already available include: numerous data retrievers from our local databases; a chromosome map summarizing genes and genomic regions linked to CHDs; pie chart generation; gene network visualization and exploration; and candidate gene prioritization. This variety of components illustrates the versatility of the approach. For instance, pie charts are easily included by calling the lightweight pieChart component with the list of slices (name and value pairs), while the prioritization 
component consists in a complete web application specifically tuned towards prioritizing CHD genes. More generally, the generic extension proved successful in the fast development of new components working as wrappers for databases, web services, command line tools, and DAS (Distributed Annotation System) servers [16]. Also, to speed up the development time for structured data updates and interaction, the extension implements a generic mechanism to easily specify web forms in Wiki text that are pre-filled and handled by registered components.

In addition, CHDWiki interacts with a patient data repository, CHDBench [15], for managing patient data published in the literature, and a DAS server [17] feeding $\mathrm{CHD}$ genes and genomic regions has been set up to allow one to loop from CHDWiki to the Ensembl genome browser and back.

When more Wiki portals such as CHDWiki are available, the problems of interoperability of these systems and integration of stored knowledge can be managed through standard protocols such as DAS for data access, web services for the programmatic use of knowledge, or dedicated application programming interfaces (APIs), which will have to be further specified by the community. The component-oriented architecture of CHDWiki will make such future developments easy to implement.

The authors had full access to the data and take responsibility for its integrity.

\section{Results}

\section{Overview of CHD data}

The results of the knowledge acquisition are described in Tables 1 and 2, and are visualized on an interactive chromosomal map in CHDWiki (Figure 1). They represent a unique repository of human genetic data for CHDs that describes both the phenotype and the genetic lesion with a granularity of detail that was unavailable so far. It allows for the addition of a free text description of any aspects of the gene that the contributor considers relevant.

For each gene or phenotype, CHDWiki provides a pie diagram that graphically represents the spectrum of related cardiac phenotypes or mutated genes, followed by a detailed overview of the studies defining the mutational spectrum of these gene-phenotype links. Moreover, to get an intuitive view of the mutation data, a graphical map of all proteins is automatically produced and updated as new genotypes are entered, displaying the position of coding mutations in the context of the protein domains. The relevance of this display is highlighted by the significant clustering of missense mutations in annotated domains (Additional file 1). More features could be added in the future (sites of protein-protein interaction or post-translational modification, for example, Figure 2).
Table 1. Number of features (genes, congenital heart defects, and so on) present in CHDWiki

\begin{tabular}{lc}
\hline Features & Entries \\
\hline Genes & 62 \\
Congenital Heart Defects & 83 \\
Linkage regions & 13 \\
Balanced chromosomal aberrations & 19 \\
Van Karnebeek and Hennekam 1999 regions & 46 \\
Indel patients & 155 \\
Indel regions & 176 \\
References & 198 \\
\hline
\end{tabular}

Table 2. Number of relations between congenital heart defects (CHD) and currently managed features

\begin{tabular}{lc}
\hline Features & CHD \\
\hline Genes & 193 \\
Linkage regions & 14 \\
Balanced chromosomal aberrations & 21 \\
Van Karnebeek and Hennekam 1999 regions & 85 \\
Patients & 281 \\
Studies (mutation screens) & 297 \\
Mutations & 284 \\
\hline
\end{tabular}

Apart from mutations detected at the nucleotide level, CHDWiki readily incorporates copy number variations and other disease-linked chromosome anomalies. Such 'chromosomal mutations' have recently been shown to be important in many disorders, including CHDs $[18,19]$.

Additionally, this portal offers a graphical overview of the protein interaction partners, as well as external links to both human and non-human genome browsers and model organism databases. Moreover, via an automated text-mining approach, genes potentially implicated in a given $\mathrm{CHD}$ and, vice versa, $\mathrm{CHDs}$ caused by a gene of interest are returned $[20,21]$. Finally, to help researchers to select candidate genes for CHDs in sets of genes (for example, identified in regions of the genome that are found through linkage analysis, homozygosity mapping or chromosomal aberrations), an adapted Endeavour algorithm for gene prioritization was implemented in CHDWiki. It offers predefined training sets of genes with tailored data sources (further details at [22]).

\section{Synthetic graphical data representation}

Associations between genes and phenotypes can be converted to networks and visualized as such. As CHDWiki is very detailed, additional features can be added to such a network (Figure 3), that is, specification of the observed gene-linked phenotypes, their frequency, the number of phenotypes shared by two genes and physical 


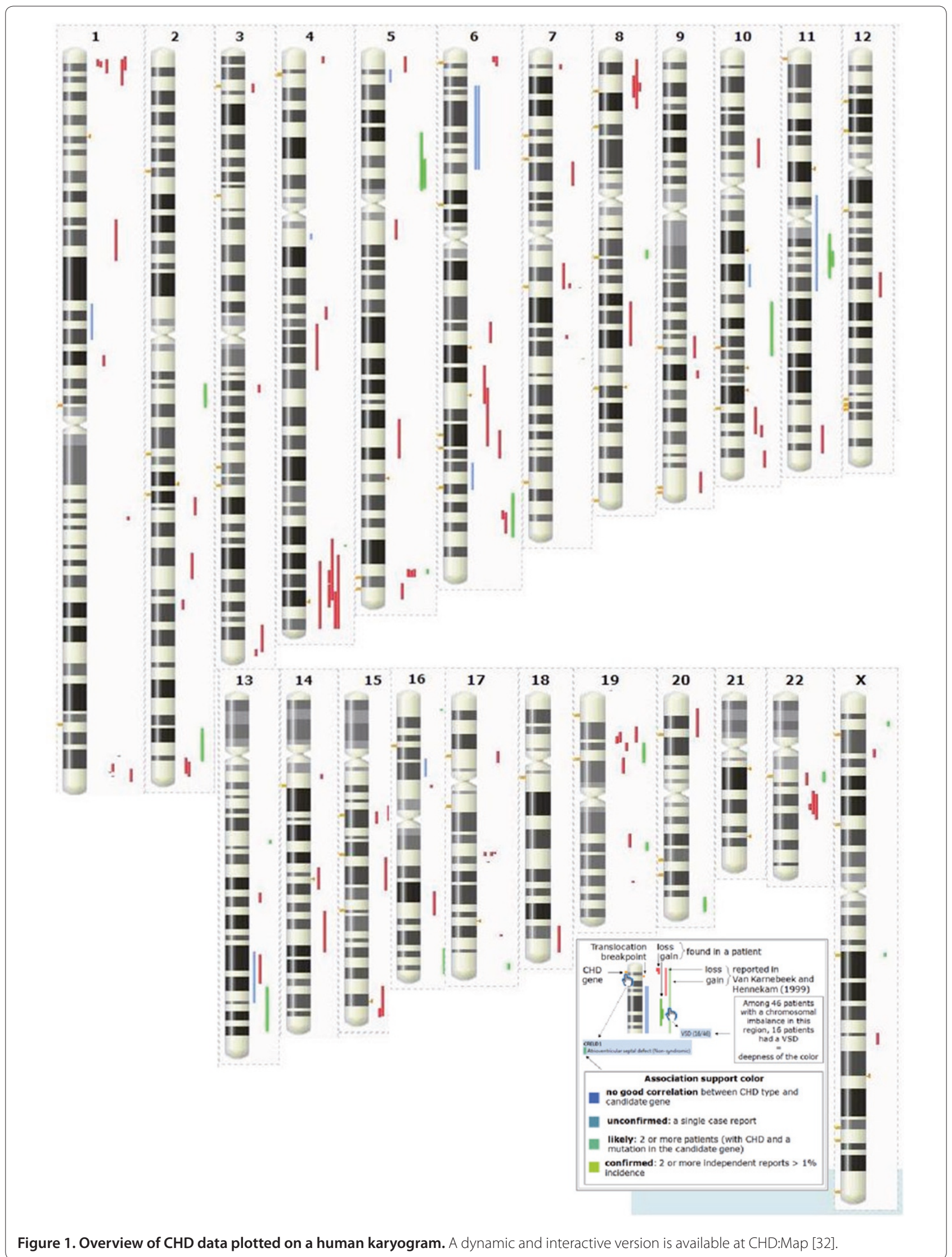




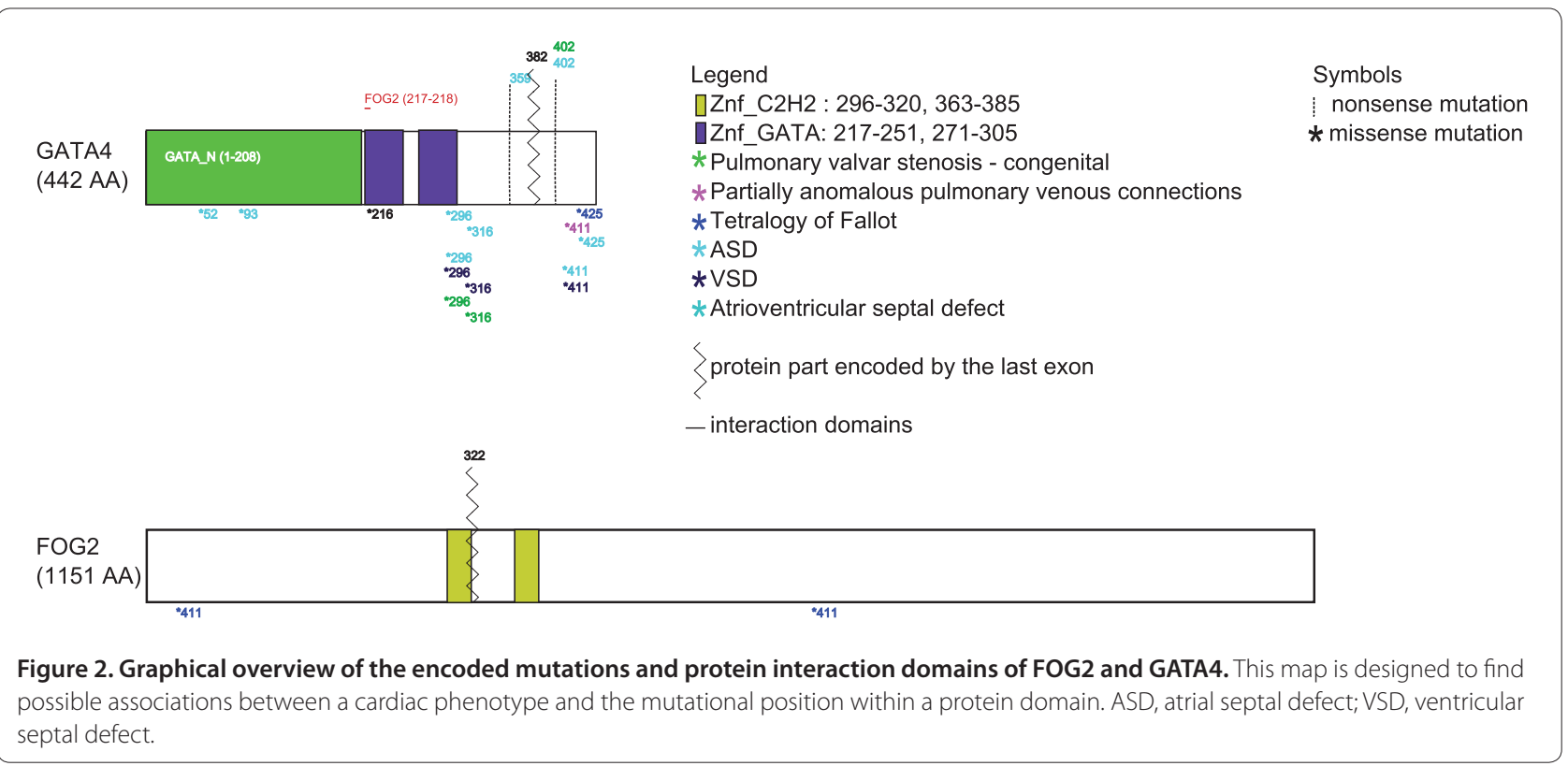

protein-protein interactions. Figure 3 is thus a synthetic representation of all data on non-syndromic CHDs. We also built similar networks for syndromic genes (Additional file 2) and networks where diseases are connected when caused by the same genes [23].

\section{Discussion \\ Wiki portals}

We argue that, next to the development of a Wikipedia for Genes, another major application of Wiki-like collaborative technology is the development of Wiki knowledge and analysis portals. In many research areas, the body of common knowledge is usually scattered across reviews, original articles, and genomic databases (MEDLINE, Ensembl and UCSC genome browsers, Entrez, OMIM, and many others). A structured Wiki allows the integration of all these data into a view that is centered on the needs of a community. A large number of bioinformatics analysis methods are available, yet a significant gap remains towards their integration within the daily practice of most biologists. By tightly integrating a knowledge base with dedicated analysis tools, a Wiki portal provides a natural stepping stone for biologists to use advanced data analysis techniques. While the development of data analysis portals has been a longstanding aim of bioinformatics, few convincing applications with a clear impact on biology have been demonstrated since the development of homology-based methods $[24,25]$. We believe that the causes of this lack of progress are mainly a lack of fine-tuning to the needs of a specific biological research area and the complexity of data analysis strategies, which appear overwhelming to biologists. We believe that Wiki portals alleviate those problems in several ways: first, by lowering the threshold towards data analysis by a tight integration with a knowledge base that is immediately useful to any biologist from the community; and second, through development of data analysis strategies sufficiently user friendly to be within reach of the majority of biologists (such as prioritization strategies, network browsing, and so on).

\section{Impact on knowledge acquisition and exchange}

In the presented CHDWiki, we compiled all information available in the literature on CHD genetics to construct a collaborative portal. We thus introduce the first example of a new type of literature review, which is dynamic and evolving; it readily provides researchers, geneticists, physicists and cardiologists with direct access to the most comprehensive knowledge on the genetics of a particular disease. An immediate impact of this methodology is how it complements and enhances the process of reviewing the literature. Wikis are clearly dynamic in nature, and can accompany a review article so that information can be kept up-to-date after the publication of the original review. A second advantage is that they cross-link interpretation and actual data: browsing such a review provides a straight link into relevant data sources (genome browsers, publications, and so on). Querying the genome on the other hand directly links into an integrated and up-to-date review. A third advantage is obviously their collaborative nature, where the expertise of numerous researchers can be pooled together to obtain extensive peer-reviewed knowledge.

The impact of disease-oriented Wikis is quite significant for both clinicians and researchers. For clinicians, it 


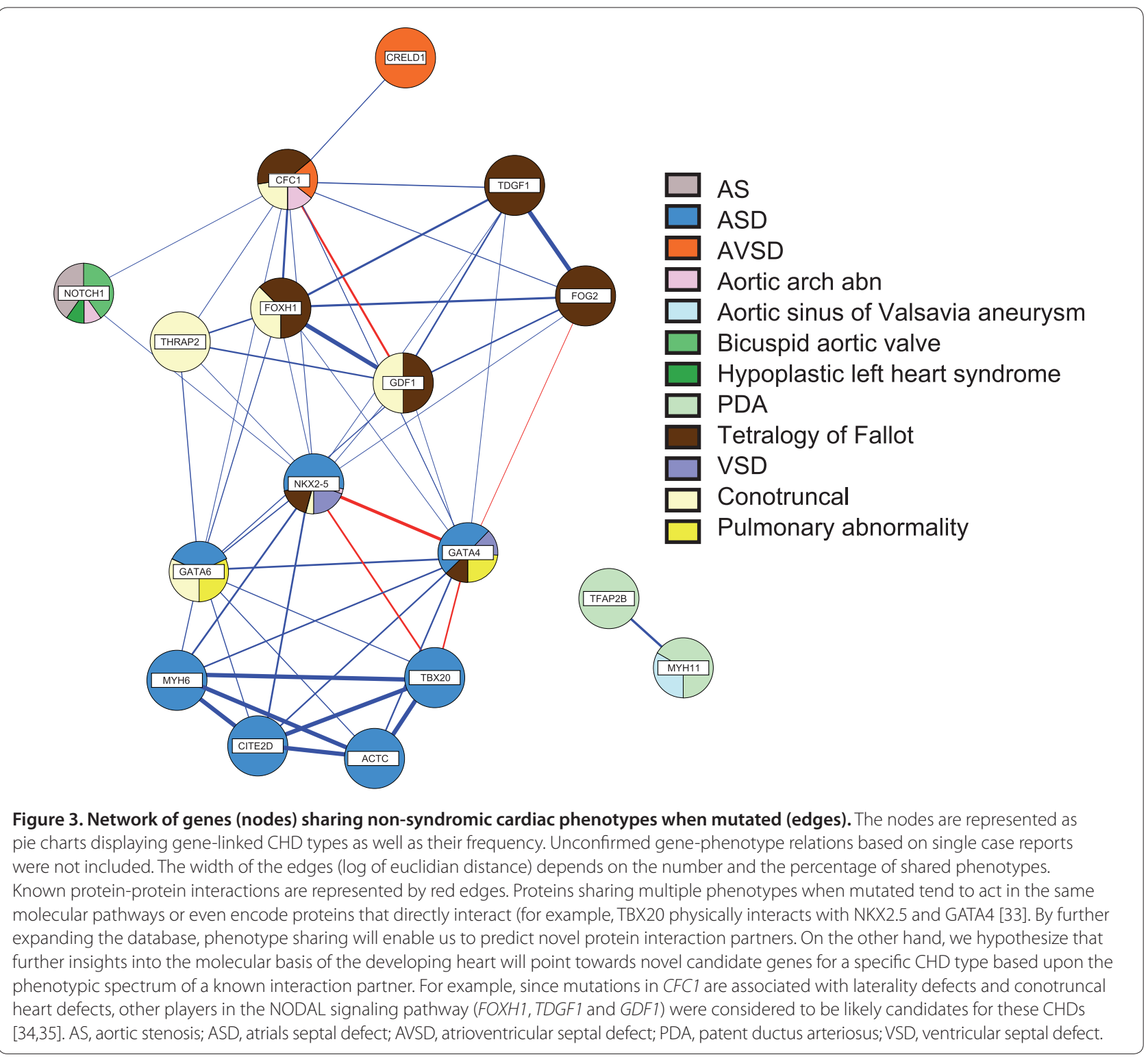

allows them to find out instantly if a gene or region has been linked to the phenotype of their patients. For instance, the finding that a chromosomal imbalance in a CHD patient affects an annotated CHD gene or region provides conclusive evidence for its causality, thus facilitating diagnosis and therapy. For the researcher, several novel regions recurrently linked to CHDs in patients emerge from the compiled genomic data, such as 1p36.3, 6q21, 15q26, 16p13.3, 17q21.3, and 22q12 (Figure 1). These provide an entry point to try to identify novel genes linked to human CHDs as illustrated [22]. Further studies are underway to obtain conclusive evidence for the involvement of these genes in CHDs, but their potential involvement is readily annotated in CHDWiki and is available to the community.
Specialized Wiki portals like CHDWiki may play a key role in the development of a universal 'Wikipedia of Genes'. Content from specialized Wikis can be compiled into a Gene Wikipedia. There might be some challenges with such integration, so an alternative model would be a federation of Wikis, where no centralized Gene Wikipedia exists, but where a hub/search engine passes queries to registered Wikis and the results are aggregated. The latter solution seems more likely since it is more efficient to develop a specialized Wiki portal, such as CHDWiki, providing specific tools for the community and focusing on a particular area. For instance, the Wikipedia page on Tetralogy of Fallot is directed towards a large audience with sections such as symptoms, diagnosis and treatments, while the CHDWiki page 
describes this phenotype succinctly and provides extensive knowledge on the genetics of this CHD (genes and mutations, patient reports). Another example is from Wikiproteins, which aims at being exhaustive about available protein knowledge, where the GATA4 entry currently links to several species and provides general information (function, localization, structure) but where its link to CHDs (ASD, Tetralogy of Fallot, and so on) is briefly and incompletely mentioned.

Wiki portals also perfectly fit with the philosophy of DASs. Not only can the data of any gene - or genomic feature - be distributed among portals and collaboratively annotated, but the genome browser can also serve as an entry point to the Wikis. CHD researchers who enable the CHDWiki tracks in a genome browser directly visualize any information compiled in CHDWiki.

\section{Collaborative aspects}

An important asset of specialized Wiki portals is that they could be a solution to the long-term maintenance of biological knowledge bases. Such long-term maintenance is a major recurring issue for the biological and bioinformatics community because of the persistent lack of long-term funding. We envisage a model for communitydriven knowledge bases where the original portal development is funded through a classical grant, but where the curation of the knowledge base is gradually shifted from the core development team to the community at large over the period of the grant (3 to 5 years). Data curation should be included in the original development cycle until a critical mass of information is reached that guarantees adoption by the community, as has been done for CHDWiki (described in the knowledge acquisition section). After curation has been shifted to the community, technical portal maintenance can be minimal.

Although such collaborative tools can easily exchange information and queries over the web, there would remain a significant risk of fragmentation if no protocols and strategies were available to effectively exchange information among the myriad of tools that are currently emerging (that is, we would have a Tower of Babel of internet-enabled tools). To satisfy the need of standardized and unified web accessible databases allowing simple data exchange, several bioinformatics initiatives have recently emerged promoting the Semantic Web (Concept Web Alliance, HCLS [26]). Indeed, the Semantic Web offers a general format for data interchange, thus providing curators with a standardized framework allowing data to be integrated and reused across disciplinesIn biology, the advantages of the Semantic Web are obvious: unique names for biological entities and consistent standards for knowledge representation, retrieval, and processing. Such standards simplify integration of web resources as they can be queried the same way.
Bioinformatics query systems in diverse fields have started to use this technology: examples include WikiPathways, a wiki dedicated to the precise collaborative annotation of metabolic pathways [27], integration of ALFRED (an allele frequency database) [28], and cancer pathways of the Starpath resource [29], and so on.

Although CHDWiki is not directly compatible with the Semantic Web approaches, it was implemented using a standard nomenclature for all terms (for example, AEPC terms for disease, EnsEMBL identifiers for genes), thus allowing straightforward transfer of its content to the new semantic web standards (for example, GEN2PHEN, a resource aimed at unifying genetic variation databases). Moreover, the CHDWiki DAS server already allows users to integrate the CHDWiki data in the EnsEMBL genome server. An important further development of our platform in this respect will be the implementation of web services following current semantic web standards. Indeed, some recent initiatives (for example, Semantic Web Mediawiki) now allow developers to combine Wiki and semantic web technologies.

Wikis also provide an effective solution to the enduring problem of unpublishable or negative results (for example, of mutation analyses). These can be highly valuable for other research teams pursuing similar paths of investigation, or contain relevant information below statistical significance. Wiki portals like CHDWiki are a natural repository for such findings. Also classic collaborative features, such as a mailing list (for example, for collaboration requests, job postings, event announcements and exchange of biological material such as DNA, cell lines, or cardiac tissue) and a directory of researchers, are basic but valuable tools such a portal can offer.

\section{Outstanding issues}

A number of technical and social issues may require significant further technology developments. The first is access control. Given the functionalities provided by a Wiki portal, it comes close to a collaborative laboratory notebook. However, research teams will understandably be reluctant to share their experimental data prior to publication. Access control restrictions (that is, deciding to provide access to part of the information to only a selected group of people) will require some further development of the Wiki platforms, although this is basically a technical problem that is routinely solved in database systems.

A second issue is quality control. Wikipedia is based on a model of peer-editing, which is generally effective but can sometimes lead to conflicts between contributors (edit wars). Such conflicts are likely to arise when conflicting scientific hypotheses and interpretations collide. Additionally, conflicts are also likely regarding scientific attribution and priority. For smaller 
Wikis, a 'benevolent dictator' who oversees any conflict can solve those problems. For larger Wikis, a hierarchy of editorial roles (contributor, reviewer, and technical, associate, and executive editors) might become necessary.

A third issue is credit assignment. Scientific credit is an essential driver and one cannot expect researchers to contribute a significant proportion of their time if no credit is attributed to them. For smaller Wikis, citation of a key publication describing the Wiki will be sufficient (exactly like for specialized databases) because key contributors will be among the authors. For larger Wikis, a direct crediting system might be difficult to establish. Significant contributions to Wikis (as contributor or editor) should be recognized as relevant scientific work and therefore appear in the track record of a researcher. Related to the issue of credit is the issue of citation. As some Wiki entries may become key scholarly references, proper citation to accurate dates or versions might be needed in scholarly work because entries change over time.

Regarding software development, each knowledge portal is likely to have highly specific aspects (type of information, source databases, ontologies or analysis tools) that make the development of a single off-the-shelf solution (similar to MediaWiki and other Wiki software) highly unlikely. A more realistic solution will lie in the development of generic tools that enable the flexible construction of such Wikis by embedding generic modules for ontology management, XML data representation, visualization, database query, and data analysis. The goal of such software will be to maximally speed up the development of Wikis while minimizing functional constraints. The current version of our Wiki framework has been made available as an open source project [30].

\section{Conclusions}

The future we envisage is one where a specialized community 'swarms' around a Wiki portal that provides most of the knowledge, data, and analysis tools needed to support its experimental work. Via this portal, the community can collaboratively and incrementally chart complex networks involved in biological processes, phenotypes, and diseases. Collaboration and efficient access to knowledge, data, and tools will significantly speed up experimental research.

\section{Availability and requirements}

CHDWiki is accessible at [31]. Data can be consulted without any registration; however, to add or modify Wiki information an account must be requested by clicking on the 'log in/create account' button at the top right of the main page.
Additional file 1. A figure of nonsense mutations encoded in the CHDWiki for the proteins NKX2-5 and TBX5. Displayed here are mutations in NKX2-5 and TBX5 present in CHDWiki. Missense mutations (asterisks) are significantly enriched in functional domains ( $P$-values: NKX2-5, $1 \times 10^{-3} ;$ TBX5, 0.05; across all nonsydromic genes, $\left.1 \times 10^{-4}\right)$. This finding is independent of the ascertainment bias associated with preferential classification of mutations affecting protein domains as pathogenic: missense mutations identified through linkage analysis in multiple individuals similarly affect preferentially protein domains ( $P$-values: NKX2-5, 0.02; TBX5, 0.05; all nonsyndromic genes combined, 0.03). This graphical representation moreover enables straightforward genotype-phenotype correlations: missense mutations causing atrial septal defects are preferentially affecting the homeobox domain ( $P$-value: $\left.1 \times 10^{-4}\right)$.

Additional file 2. A figure showing a network with genes sharing syndromic cardiac phenotypes when mutated. Network with genes (nodes) sharing syndromic cardiac phenotypes when mutated (edges). As members of the RAS-MAP kinase pathway (PTPN11, SOS1, BRAF, KRAS, MAP2K1, MAP2K2, SHOC2 and NF1) clearly form a phenotypic cluster, they seem to be involved in the same developmental cardiac cell lineages.

\section{Abbreviations}

AEPC, Association for European Paediatric Cardiology; CHD, congenital heart defect; DAS, Distributed Annotation System.

\section{Competing interests}

The authors declare that they have no competing interests.

\section{Authors' contributions}

$\mathrm{RB}, \mathrm{JB}, \mathrm{BT}$ and SB participated in the design of the study and drafted the manuscript. KD and YM conceived of the study, participated in its design and coordination, and drafted the manuscript. All co-authors contributed to various aspects of the platform development: Wiki development (RB, SB, YM), Gene networks (RB, SB), genotype-phenotype relations (JB, BT, KD), Bench $(\mathrm{SW}, \mathrm{BC})$, and gene prioritization tools ( $L C T, P V L)$. Knowledge acquisition was performed by JB, BT, KD and MG. All the co-authors listed above fulfill the three requirements for authorship defined by the ICMJE guidelines.

\section{Acknowledgements}

We gratefully acknowledge Professor JE Lock (Cardiovascular program, Department of Cardiology, Children's Hospital Boston, MA, USA) for providing cartoons of heart defects. This research was supported by the EU FP7 CheartED project (Grant HEALTH-F2-2008-223040). Other grants were provided by the Research Council KUL (GOA MaNet, GOA AMBioRICS, CoE EF/05/007 SymBioSys, PROMETA, START 1, several PhD/postdoc \& fellow grants, GOA 2006/12), FWO (PhD/postdoc grants, projects G.0241.04 (Functional Genomics), G.0499.04 (Statistics), G.0232.05 (Cardiovascular), G.0318.05 (subfunctionalization), G.0553.06 (VitamineD), G.0302.07 (SVM/ Kernel), research communities (ICCOS, ANMMM, MLDM)), G.0733.09 3UTR; G. 082409 (EGFR), G.0254.05 (Genetics of human heart development), IWT (PhD Grants, GBOU-McKnow-E (Knowledge management algorithms), GBOU-ANA (Biosensors), TAD-BioScope-IT, Silicos; SBO-BioFrame, SBO-MoKa, TBM Endometriosis), the Belgian Federal Science Policy Office (IUAP P6/25 (BioMaGNet, Bioinformatics and Modeling: from Genomes to Networks, 2007-2011), IUAP P5/25 (Molecular Pathology of Genetic Diseases)) and the EU-RTD (ERNSI: European Research Network on System Identification; FP6-NoE Biopattern; FP6-IP e-Tumours, FP6-MC-EST Bioptrain, FP6-STREP Strokemap). PVL is supported by a postdoctoral research fellowship, JB by a PhD fellowship and $\mathrm{KD}$ is a senior clinical investigator of the Research Foundation-Flanders (FWO). SB is supported by a post-doc grant of the CheartED project.

\section{Author details}

'Bioinformatics Group, Department of Electrical Engineering, ESAT-SCD, Katholieke Universiteit Leuven, Kasteelpark Arenberg 10, B-3001 Leuven, Belgium. ${ }^{2}$ Université de Toulouse, UPS, Laboratoire de Microbiologie et 
Génétique Moléculaires, 118 route de Narbonne, F-31000 Toulouse, France. ${ }^{3}$ Centre National de la Recherche Scientifique, LMGM, 118 route de Narbonne, F-31000 Toulouse, France. ${ }^{4}$ Center for Human Genetics, University Hospital Leuven, Herestraat 49, B-3000 Leuven, Belgium. ${ }^{5}$ Laboratory of Molecular Signalling and Laboratory of Developmental Genetics and Imprinting, Babraham Research Campus, Cambridge CB22 3AT, United Kingdom. ${ }^{6}$ Department of Molecular and Developmental Genetics, VIB, Herestraat 49, B-3000, Leuven, Belgium. 'Department of Pediatric Cardiology, University Hospital Leuven, Herestraat 49, B-3000 Leuven, Belgium.

Received: 18 November 2009 Revised: 15 January 2010

Accepted: 1 March 2010 Published: 1 March 2010

\section{References}

1. Mons B, Ashburner M, Chichester C, van Mulligen E, Weeber M, den Dunnen J, van Ommen GJ, Musen M, Cockerill M, Hermjakob H, Mons A, Packer A, Pacheco R, Lewis S, Berkeley A, Melton W, Barris N, Wales J, Meijssen G, Moeller E, Roes PJ, Borner K, Bairoch A: Calling on a million minds for community annotation in WikiProteins. Genome Biol 2008, 9:R89.

2. Huss JW 3rd, Orozco C, Goodale J, Wu C, Batalov S, Vickers TJ, Valafar F, Su Al: A gene wiki for community annotation of gene function. PLOS Biol 2008, 6:e175.

3. Hu JC, Aramayo R, Bolser D, Conway T, Elsik CG, Gribskov M, Kelder T, Kihara D, Knight TF Jr, Pico AR, Siegele DA, Wanner BL, Welch RD: The emerging world of wikis. Science 2008, 320:1289-1290

4. Wang K: Gene-function wiki would let biologists pool worldwide resources. Nature 2006, 439:534.

5. Giles J: Key biology databases go wiki. Nature 2007, 445:691.

6. Hoffmann R: A wiki for the life sciences where authorship matters. Nat Genet 2008, 40:1047-1051.

7. Kohler S, Bauer S, Horn D, Robinson PN: Walking the interactome for prioritization of candidate disease genes. Am J Hum Genet 2008, 82:949-958.

8. Lage K, Karlberg EO, Storling ZM, Olason PI, Pedersen AG, Rigina O, Hinsby AM, Tumer Z, Pociot F, Tommerup N, Moreau Y, Brunak S: A human phenome-interactome network of protein complexes implicated in genetic disorders. Nat Biotechnol 2007, 25:309-316.

9. Aerts S, Lambrechts D, Maity S, Van Loo P, Coessens B, De Smet F, Tranchevent LC, De Moor B, Marynen P, Hassan B, Carmeliet P, Moreau Y: Gene prioritization through genomic data fusion. Nat Biotechnol 2006, 24:537-544.

10. Goh KI, Cusick ME, Valle D, Childs B, Vidal M, Barabasi AL: The human disease network. Proc Natl Acad Sci U S A 2007, 104:8685-8690

11. Schuster-Bockler B, Bateman A: Protein interactions in human genetic diseases. Genome Biol 2008, 9:R9.

12. McGary KL, Lee I, Marcotte EM: Broad network-based predictability of Saccharomyces cerevisiae gene loss-of-function phenotypes. Genome Biol 2007, 8:R258.

13. CHD:Review [http://www.esat.kuleuven.be/ bioiuser/chdwiki/index.php/ CHD:Review]

14. van Karnebeek CD, Hennekam RC: Associations between chromosomal anomalies and congenital heart defects: a database search. Am J Med Genet 1999, 84:158-166.

15. CHDBench [http://tomcat.esat.kuleuven.be/chdbench]

16. Prlic A, Down TA, Kulesha E, Finn RD, Kahari A, Hubbard TJ: Integrating sequence and structural biology with DAS. BMC Bioinformatics 2007, 8:333.

17. Finn RD, Stalker JW, Jackson DK, Kulesha E, Clements J, Pettett R: ProServer: a simple, extensible Perl DAS server. Bioinformatics 2007, 23:1568-1570.

18. Thienpont B, Mertens L, de Ravel T, Eyskens B, Boshoff D, Maas N, Fryns JP, Gewillig M, Vermeesch JR, Devriendt K: Submicroscopic chromosomal imbalances detected by array-CGH are a frequent cause of congenital heart defects in selected patients. Eur Heart J 2007, 28:2778-2784.

19. Greenway SC, Pereira AC, Lin JC, DePalma SR, Israel SJ, Mesquita SM, Ergul E, Conta JH, Korn JM, McCarroll SA, Gorham JM, Gabriel S, Altshuler DM, Quintanilla-Dieck Mde L, Artunduaga MA, Eavey RD, Plenge RM, Shadick NA, Weinblatt ME, De Jager PL, Hafler DA, Breitbart RE, Seidman JG, Seidman CE: De novo copy number variants identify new genes and loci in isolated sporadic tetralogy of Fallot. Nat Genet 2009, 41:931-935.

20. Van Vooren S, Thienpont B, Menten B, Speleman F, De Moor B, Vermeesch J, Moreau Y: Mapping biomedical concepts onto the human genome by mining literature on chromosomal aberrations. Nucleic Acids Res 2007, 35:2533-2543

21. Yu W, Gwinn M, Clyne M, Yesupriya A, Khoury MJ: A navigator for human genome epidemiology. Nat Genet 2008, 40:124-125.

22. CHD: Gene hunting example [http://homes.esat.kuleuven.be/ bioiuser/ chdwiki/index.php/CHD:Gene_hunting_example]

23. CHD gene networks [http://homes.esat.kuleuven.be/ bioiuser/chdwiki/ index.php/CHD_gene_networks]

24. Altschul SF, Gish W, Miller W, Myers EW, Lipman DJ: Basic local alignment search tool. J Mol Biol 1990, 215:403-410.

25. Mulder NJ, Apweiler R: The InterPro database and tools for protein domain analysis. Curr Protoc Bioinformatics 2008, Chapter 2:Unit 27.

26. Cheung KH, Yip KY, Townsend JP, Scotch M: HCLS 2.0/3.0: health care and life sciences data mashup using Web 2.0/3.0. J Biomed Inform 2008, 41:694-705

27. Pico AR, Kelder T, van lersel MP, Hanspers K, Conklin BR, Evelo C: WikiPathways: pathway editing for the people. PLoS Biol 2008, 6:e184.

28. Rajeevan H, Cheung KH, Gadagkar R, Stein S, Soundararajan U, Kidd JR, Pakstis AJ, Miller PL, Kidd KK: ALFRED: An Allele Frequency Database for Microevolutionary Studies. Evol Bioinform Online 2005, 1:1-10.

29. Holford ME, Rajeevan H, Zhao H, Kidd KK, Cheung KH: Semantic web-based integration of cancer pathways and allele frequency data. Cancer Inform 2009, 8:19-30.

30. Extension:WikiOpener [http://www.mediawiki.org/wiki/ Extension:WikiOpener]

31. CHDWiki [http://www.esat.kuleuven.be/ bioiuser/chdwiki]

32. CHD:Map [http://www.esat.kuleuven.be/ bioiuser/chdwiki/index.php/ CHD:Map]

33. Stennard FA, Costa MW, Elliott DA, Rankin S, Haast SJ, Lai D, McDonald LP, Niederreither K, Dolle P, Bruneau BG, Zorn AM, Harvey RP: Cardiac T-box factor Tbx20 directly interacts with Nkx2-5, GATA4, and GATA5 in regulation of gene expression in the developing heart. Dev Biol 2003, 262:206-224.

34. Karkera JD, Lee JS, Roessler E, Banerjee-Basu S, Ouspenskaia MV, Mez J, Goldmuntz E, Bowers P, Towbin J, Belmont JW, Baxevanis AD, Schier AF Muenke M: Loss-of-function mutations in growth differentiation factor-1 (GDF1) are associated with congenital heart defects in humans. Am J Hum Genet 2007, 81:987-994

35. Roessler E, Ouspenskaia MV, Karkera JD, Velez JI, Kantipong A, Lacbawan F, Bowers P, Belmont JW, Towbin JA, Goldmuntz E, Feldman B, Muenke M: Reduced NODAL signaling strength via mutation of several pathway members including FOXH1 is linked to human heart defects and holoprosencephaly. Am J Hum Genet 2008, 83:18-29.

doi:10.1186/gm137

Cite this article as: Barriot $\mathrm{R}$, et al.: Collaboratively charting the gene-tophenotype network of human congenital heart defects. Genome Medicine 2010, 2:16. 